\title{
Some inequalities for unitarily invariant norms of matrices
}

\author{
Shaoheng Wang, Limin Zou* and Youyi Jiang
}

* Correspondence: limin-zou@163. com

School of Mathematics and Statistics, Chongqing Three Gorges University, Chongqing, 404000,

People's Republic of China

\section{Abstract}

This article aims to discuss inequalities involving unitarily invariant norms. We obtain a refinement of the inequality shown by Zhan. Meanwhile, we give an improvement of the inequality presented by Bhatia and Kittaneh for the Hilbert-Schmidt norm.

Mathematical Subject Classification: MSC (2010) 15A60; 47A30; 47B15

Keywords: Unitarily invariant norms, Positive semidefinite matrices, Convex function, Inequality

\section{Introduction}

Let $M_{m, n}$ be the space of $m \times n$ complex matrices and $M_{n}=M_{n, n}$. Let $\|\cdot\|$ denote any unitarily invariant norm on $M_{n}$. So, $\|U A V\|=\|A\|$ for all $A \in M_{n}$ and for all unitary matrices $U, V \in M_{n}$. For $A=\left(a_{i j}\right) \in M_{n}$, the Hilbert-Schmidt norm of $A$ is defined by

$$
\|A\|_{2}=\sqrt{\left(\sum_{i=1}^{n} \sum_{j=1}^{n}\left|a_{i j}\right|^{2}\right)}=\sqrt{t r|A|^{2}}=\sqrt{\sum_{j=1}^{n} s_{j}^{2}(A)},
$$

where $\operatorname{tr}$ is the usual trace functional and $s_{1}(A) \geq s_{2}(A) \geq \ldots \geq s_{n-1}(A) \geq s_{n}(A)$ are the singular values of $A$, that is, the eigenvalues of the positive semidefinite matrix $|A|=\left(A A^{*}\right) \frac{1}{2}$, arranged in decreasing order and repeated according to multiplicity. The Hilbert-Schmidt norm is in the class of Schatten norms. For $1 \leq p<\alpha$, the Schatten $p$ norm $\|\cdot\|_{p}$ is defined as

$$
\|A\|_{p}=\left(\sum_{j=1}^{n} s_{j}^{p}(A)\right)^{1 / p}=\left(\operatorname{tr}|A|^{p}\right)^{1 / p} .
$$

For $k=1, \ldots, n$, the $K y$ Fan $k$-norm $\|\cdot\|_{(k)}$ is defined as

$$
\|A\|_{(k)}=\sum_{j=1}^{k} s_{j}(A)
$$

It is known that these norms are unitarily invariant, and it is evident that each unitarily invariant norm is a symmetric guage function of singular values [1, p. 54-55].

Bhatia and Davis proved in [2] that if $A, B, X \in M_{n}$ such that $A$ and $B$ are positive semidefinite and if $0 \leq r \leq 1$, then

\section{SpringerOpen ${ }^{\circ}$}

(C) 2011 Wang et al; licensee Springer. This is an Open Access article distributed under the terms of the Creative Commons Attribution License (http://creativecommons.org/licenses/by/2.0), which permits unrestricted use, distribution, and reproduction in any medium, provided the original work is properly cited. 


$$
2\left\|A^{1 / 2} X B^{1 / 2}\right\| \leq\left\|A^{r} X B^{1-r}+A^{1-r} X B^{r}\right\| \leq\|A X+X B\|
$$

Let $A, B, X \in M_{n}$ such that $A$ and $B$ are positive semidefinite. In [3], Zhan proved that

$$
\left\|A^{r} X B^{2-r}+A^{2-r} X B^{r}\right\| \leq \frac{2}{t+2}\left\|A^{2} X+t A X B+X B^{2}\right\|
$$

for any unitarily invariant norm and real numbers $r, t$ satisfying $1 \leq 2 r \leq 3,-2<t \leq 2$. The case $r=1, t=0$ of this result is the well-known arithmetic-geometric mean inequality

$$
2\left\|A^{1 / 2} X B^{1 / 2}\right\| \leq\|A X+X B\| .
$$

Meanwhile, for $r \in[0,1]$, Zhan pointed out that he can get another proof of the following well-known Heinz inequality

$$
\left\|A^{r} X B^{1-r}+A^{1-r} X B^{r}\right\| \leqslant\|A X+X B\|
$$

by the same method used in the proof of (1.2).

Let $A, B, X \in M_{n}$ such that $A$ and $B$ are positive semidefinite and suppose that

$$
\psi(v)=\left\|A^{1+v} X B^{1-v}+A^{1-v} X B^{1+v}\right\|
$$

Then $\psi$ is a convex function on $[-1,1]$ and attains its minimum at $v=0$ [4, p. 265]. In [5], for positive semidefinite $n \times n$ matrices, the inequality

$$
\|A B\| \leqslant \frac{1}{4}\left\|(A+B)^{2}\right\|
$$

was shown to hold for every unitarily invariant norm. Meanwhile, Bhatia and Kittaneh [5] asked the following.

\section{Question}

Let $A, B \in M_{n}$ be positive semidefinite. Is it true that

$$
s_{j}(A B) \leqslant \frac{1}{4} s_{j}(A+B)^{2}, j=1,2, \cdots, n ?
$$

The case $n=2$ is known to be true [5]. (See also, [1, p. 133], [6, p. 2189-2190], [7, p. 198].)

Obviously, if $A, B \in M_{n}$ are positive semidefinite and $A B=B A$, then we have $j=1,2, \cdots, n, j=1,2, \cdots, n$.

\section{Some inequalities for unitarily invariant norms}

In this section, we first utilize the convexity of the function

$$
\psi(r)=\left\|A^{r} X B^{2-r}+A^{2-r} X B^{r}\right\|
$$

to obtain an inequality for unitarily invariant norms that leads to a refinement of the inequality (1.2). To do this, we need the following lemmas on convex functions.

\section{Lemma 2.1}

Let $A, B, X \in M_{n}$ such that $A$ and $B$ are positive semidefinite. Then, for each unitarily invariant norm, the function 


$$
\psi(r)=\left\|A^{r} X B^{2-r}+A^{2-r} X B^{r}\right\|
$$

is convex on $[0,2]$ and attains its minimum at $r=1$.

\section{Proof}

Replace $v+1$ by $r$ in (1.3). $\square$

\section{Lemma 2.2}

Let $\psi$ be a real valued convex function on an interval $[a, b]$ which contains $\left(x_{1}, x_{2}\right)$. Then for $x_{1} \leq x \leq x_{2}$, we have

$$
\psi(x) \leq \frac{\psi\left(x_{2}\right)-\psi\left(x_{1}\right)}{x_{2}-x_{1}} x-\frac{x_{1} \psi\left(x_{2}\right)-x_{2} \psi\left(x_{1}\right)}{x_{2}-x_{1}} .
$$

Proof

Since $\psi$ is a convex function on $[a, b]$, for $a \leq x_{1} \leq x \leq x_{2} \leq b$, we have

$$
\frac{\psi\left(x_{1}\right)-\psi(x)}{x_{1}-x} \leq \frac{\psi\left(x_{2}\right)-\psi(x)}{x_{2}-x} .
$$

This is equivalent to the inequality (2.1). $\square$

\section{Theorem 2.1}

Let $A, B, X \in M_{n}$ such that $A$ and $B$ are positive semidefinite. If $1 \leq 2 r \leq 3$ and $-2<t \leq 2$, then

$$
\left\|A^{r} X B^{2-r}+A^{2-r} X B^{r}\right\| \leq 2\left(2 r_{0}-1\right)\|A X B\|+\frac{4\left(1-r_{0}\right)}{2+t}\left\|A^{2} X+t A X B+X B^{2}\right\|,
$$

where $r_{0}=\min \{r, 2-r\}$.

\section{Proof}

If $\frac{1}{2} \leqslant r \leqslant 1$, then by Lemma 2.1 and Lemma 2.2 , we have

$$
\psi(r) \leq \frac{\psi(1)-\psi\left(\frac{1}{2}\right)}{1-\frac{1}{2}} r-\frac{\frac{1}{2} \psi(1)-\psi\left(\frac{1}{2}\right)}{1-\frac{1}{2}}
$$

That is

$$
\psi(r) \leq(2 r-1) \psi(1)+2(1-r) \psi\left(\frac{1}{2}\right)
$$

It follows from (1.2) and (2.3) that

$$
\left\|A^{r} X B^{2-r}+A^{2-r} X B^{r}\right\| \leq 2(2 r-1)\|A X B\|+\frac{4(1-r)}{2+t}\left\|A^{2} X+t A X B+X B^{2}\right\| .
$$


If $1 \leqslant r \leqslant \frac{3}{2}$, then by Lemma 2.1 and Lemma 2.2, we have

$$
\psi(r) \leq \frac{\psi\left(\frac{3}{2}\right)-\psi(1)}{\frac{3}{2}-1} r-\frac{\psi\left(\frac{3}{2}\right)-\frac{3}{2} \psi(1)}{\frac{3}{2}-1}
$$

That is

$$
\psi(r) \leq(3-2 r) \psi(1)+2(r-1) \psi\left(\frac{3}{2}\right) .
$$

It follows from (1.2) and (2.4) that

$$
\left\|A^{r} X B^{2-r}+A^{2-r} X B^{r}\right\| \leq 2(3-2 r)\|A X B\|+\frac{4(r-1)}{2+t}\left\|A^{2} X+t A X B+X B^{2}\right\| .
$$

It is equivalent to the following inequality

$$
\left\|A^{r} X B^{2-r}+A^{2-r} X B^{r}\right\| \leq 2\left(2 r_{0}-1\right)\|A X B\|+\frac{4\left(1-r_{0}\right)}{2+t}\left\|A^{2} X+t A X B+X B^{2}\right\| .
$$

This completes the proof..

Now, we give a simple comparison between the upper bound in (1.2) and the upper bound in (2.2).

$$
\begin{aligned}
& \frac{2}{2+t}\left\|A^{2} X+t A X B+X B^{2}\right\|-2\left(2 r_{0}-1\right)\|A X B\|-\frac{4\left(1-r_{0}\right)}{2+t}\left\|A^{2} X+t A X B+X B^{2}\right\| \\
& =\frac{2\left(2 r_{0}-1\right)}{2+t}\left\|A^{2} X+t A X B+X B^{2}\right\|-2\left(2 r_{0}-1\right)\|A X B\| \\
& \geq \frac{2\left(2 r_{0}-1\right)}{2+t} \cdot(2+t)\|A X B\|-2\left(2 r_{0}-1\right)\|A X B\|=0 .
\end{aligned}
$$

Therefore, Theorem 2.1 is a refinement of the inequality (1.2).

Let $A, B, X \in M_{n}$ such that $A$ and $B$ are positive semidefinite. Then, for each unitarily invariant norm, the function

$$
\varphi(v)=\left\|A^{v} X B^{1-v}+A^{1-v} X B^{v}\right\|
$$

is a continuous convex function on $[0,1]$ and attains its minimum at $v=\frac{1}{2}$. See $[4, \mathrm{p}$.

265]. Then, by the same method above, we have the following result.

\section{Theorem 2.2.[8]}

Let $A, B, X \in M_{n}$ such that $A$ and $B$ are positive semidefinite. If $0 \leq v \leq 1$, then

$$
\left\|A^{v} X B^{1-v}+A^{1-v} X B^{v}\right\| \leq 4 r_{0}\left\|A^{1 / 2} X B^{1 / 2}\right\|+\left(1-2 r_{0}\right)\|A X+X B\|,
$$

where $r_{0}=\min \{v, 1-v\}$. This is a refinement of the second inequality in (1.1).

Next, we will obtain an improvement of the inequality (1.4) for the Hilbert-Schmidt norm. To do this, we need the following lemma. 


\section{Lemma 2.3.[9]}

Let $A, B, X \in M_{n}$ such that $A$ and $B$ are positive semidefinite. If $0 \leq v \leq 1$, then

$$
\left\|A^{v} X B^{1-v}\right\| \leq\|A X\|^{v}\|X B\|^{1-v} .
$$

\section{Theorem 2.3}

Let $A, B, X \in M_{n}$ such that $A$ and $B$ are positive semidefinite. If $0 \leq v \leq 1$, then

$$
2\left\|A^{v} X B^{1-v}\right\|+\left(\|A X\|^{v}-\|X B\|^{1-v}\right)^{2} \leq \sqrt{\|A X\|^{4 v}+\|X B\|^{4(1-v)}+2\left\|A^{v} X B^{1-v}\right\|^{2}} .
$$

\section{Proof}

Let

$$
S=\|A X\|^{4 v}+\|X B\|^{4(1-v)}+2\left\|A^{v} X B^{1-v}\right\|^{2}-\left(2\left\|A^{v} X B^{1-v}\right\|+\left(\|A X\|^{v}-\|X B\|^{1-v}\right)^{2}\right)^{2} .
$$

So,

$$
\begin{aligned}
& S=\|A X\|^{4 v}+\|X B\|^{4(1-v)}+2\left\|A^{v} X B^{1-v}\right\|^{2}-4\left\|A^{v} X B^{1-v}\right\|^{2}-\left(\|A X\|^{v}-\|X B\|^{1-v}\right)^{4} \\
& -4\left\|A^{v} X B^{1-v}\right\|\left(\|A X\|^{v}-\|X B\|^{1-v}\right)^{2} \\
& =\|A X\|^{4 v}+\|X B\|^{4(1-v)}-2\left\|A^{v} X B^{1-v}\right\|^{2}-\left(\|A X\|^{v}-\|X B\|^{1-v}\right)^{4} \\
& -4\left\|A^{v} X B^{1-v}\right\|\left(\|A X\|^{v}-\|X B\|^{1-v}\right)^{2} .
\end{aligned}
$$

By Lemma 2.3, we have

$$
\begin{aligned}
& S \geq\|A X\|^{4 v}+\|X B\|^{4(1-v)}-2\|A X\|^{2 v}\|X B\|^{2(1-v)}-\left(\|A X\|^{v}-\|X B\|^{1-v}\right)^{4} \\
& -4\left\|A^{v} X B^{1-v}\right\|\left(\|A X\|^{v}-\|X B\|^{1-v}\right)^{2} .
\end{aligned}
$$

That is,

$$
\begin{aligned}
& S \geq\left(\|A X\|^{v}-\|X B\|^{1-v}\right)^{2}\left(\left(\|A X\|^{v}+\|X B\|^{1-v}\right)^{2}-\left(\|A X\|^{v}-\|X B\|^{1-v}\right)^{2}-4\left\|A^{v} X B^{1-v}\right\|\right) \\
& =4\left(\|A X\|^{v}-\|X B\|^{1-v}\right)^{2}\left(\|A X\|^{v}\|X B\|^{1-v}-\left\|A^{v} X B^{1-v}\right\|\right) \\
& \geq 0 .
\end{aligned}
$$

Hence,

$$
\|A X\|^{4 v}+\|X B\|^{4(1-v)}+2\left\|A^{v} X B^{1-v}\right\|^{2} \geq\left(2\left\|A^{v} X B^{1-v}\right\|+\left(\|A X\|^{v}-\|X B\|^{1-v}\right)^{2}\right)^{2} .
$$

This completes the proof. $\square$

Let $A, B, X \in M_{n}$ such that $A$ and $B$ are positive semidefinite, for Hilbert-Schmidt norm, the following equality holds:

$$
\|A X+X B\|_{2}^{2}=\|A X\|_{2}^{2}+\|X B\|_{2}^{2}+2\left\|A^{1 / 2} X B^{1 / 2}\right\|_{2}^{2} .
$$

Taking $v=\frac{1}{2}$ in Theorem 2.3, and then we have the following result. 
Theorem 2.4.[10]

Let $A, B, X \in M_{n}$ such that $A$ and $B$ are positive semidefinite. Then

$$
2\left\|A^{1 / 2} X B^{1 / 2}\right\|_{2}+\left(\sqrt{\|A X\|_{2}}-\sqrt{\|X B\|_{2}}\right)^{2} \leq\|A X+X B\|_{2} .
$$

Bhatia and Kittaneh proved in [5] that if $A, B \in M_{n}$ are positive semidefinite, then

$$
\left\|A^{3 / 2} B^{1 / 2}+A^{1 / 2} B^{3 / 2}\right\| \leq \frac{1}{2}\left\|(A+B)^{2}\right\|
$$

Now, we give an improvement of the inequality (1.4) for the Hilbert-Schmidt norm.

\section{Theorem 2.5}

Let $A, B \in M_{n}$ be positive semidefinite. Then

$$
\|A B\|_{2}+\frac{1}{2}\left(\sqrt{\left\|A^{3 / 2} B^{1 / 2}\right\|_{2}}-\sqrt{\left\|A^{1 / 2} B^{3 / 2}\right\|_{2}}\right)^{2} \leq \frac{1}{4}\left\|(A+B)^{2}\right\|_{2} .
$$

Proof

Let

$$
X=A^{1 / 2} B^{1 / 2} .
$$

Then, by Theorem 2.4, we have

$$
2\|A B\|_{2}+\left(\sqrt{\left\|A^{3 / 2} B^{1 / 2}\right\|_{2}}-\sqrt{\left\|A^{1 / 2} B^{3 / 2}\right\|_{2}}\right)^{2} \leq\left\|A^{3 / 2} B^{1 / 2}+A^{1 / 2} B^{3 / 2}\right\|_{2} .
$$

It follows form (2.5) and (2.6) that

$$
2\|A B\|_{2}+\left(\sqrt{\left\|A^{3 / 2} B^{1 / 2}\right\|_{2}}-\sqrt{\left\|A^{1 / 2} B^{3 / 2}\right\|_{2}}\right)^{2} \leq \frac{1}{2}\left\|(A+B)^{2}\right\|_{2} .
$$

That is,

$$
\|A B\|_{2}+\frac{1}{2}\left(\sqrt{\left\|A^{3 / 2} B^{1 / 2}\right\|_{2}}-\sqrt{\left\|A^{1 / 2} B^{3 / 2}\right\|_{2}}\right)^{2} \leq \frac{1}{4}\left\|(A+B)^{2}\right\|_{2} .
$$

This completes the proof. $\square$

\section{Acknowledgements}

The authors wish to express their heartfelt thanks to the referees and Professor Vijay Gupta for their detailed and helpful suggestions for revising the manuscript. At the same time, we are grateful for the suggestions of Yang Peng. This research was supported by Natural Science Foundation Project of Chongqing Science and Technology

Commission (No. CSTC, 2010BB0314), Natural Science Foundation of Chongqing Municipal Education Commission (No. KJ101108), and Scientific Research Project of Chongqing Three Gorges University (No. 10ZD-16).

\section{Authors' contributions}

SW and LZ designed and performed all the steps of proof in this research and also wrote the paper. YJ participated in the design of the study and suggest many good ideas that made this paper possible and helped to draft the first manuscript. All authors read and approved the final manuscript.

\section{Competing interests}

The authors declare that they have no competing interests. 


\section{References}

1. Zhan, X: Matrix Theory. Higher Education Press, Beijing (2008) (in Chinese)

2. Bhatia, R, Davis, C: More matrix forms of the arithmetic-geometric mean inequality. SIAM J Matrix Anal Appl. 14 132-136 (1993). doi:10.1137/0614012

3. Zhan, X: Inequalities for unitarily invariant norms. SIAM J Matrix Anal Appl. 20, 466-470 (1998). doi:10.1137/ S0895479898323823

4. Bhatia, R: Matrix Analysis. Springer-Verlag, New York (1997)

5. Bhatia, R, Kittaneh, F: Notes on matrix arithmetic-geometric mean inequalities. Linear Algebra Appl. 308, 203-211 (2000). doi:10.1016/S0024-3795(00)00048-3

6. Bhatia, R, Kittaneh, F: The matrix arithmetic-geometric mean inequality revisited. Linear Algebra Appl. 428, $2177-2191$ (2008)

7. Bhatia, R: Positive Definite Matrices. Princeton University Press, Princeton (2007)

8. Kittaneh, F: On the convexity of the Heinz means. Integr Equ Oper Theory. 68, 519-527 (2010). doi:10.1007/s00020-0101807-6

9. Kittaneh, F: Norm inequalities for fractional powers of positive operators. Lett Math Phys. 27, 279-285 (1993). doi:10.1007/BF00777375

10. Kittaneh, F, Manasrah, Y: Improved Young and Heinz inequalities for matrices. J Math Anal Appl. 361, $262-269$ (2010). doi:10.1016/j.jmaa.2009.08.059

doi:10.1186/1029-242X-2011-10

Cite this article as: Wang et al:: Some inequalities for unitarily invariant norms of matrices. Journal of Inequalities and Applications 2011 2011:10.

\section{Submit your manuscript to a SpringerOpen ${ }^{\circ}$} journal and benefit from:

- Convenient online submission

- Rigorous peer review

- Immediate publication on acceptance

- Open access: articles freely available online

- High visibility within the field

- Retaining the copyright to your article

Submit your next manuscript at $>$ springeropen.com 